\title{
Biomarkers and prevalence of cardiometabolic syndrome among people living with HIV/AIDS, Addis Ababa, Ethiopia: a hospital- based, observational study.
}

\author{
Minyahil Woldu ( $\square$ minyahil.alebachew@aau.edu.et) \\ Muhimbili University of Health and Allied Sciences \\ Omary Minzi \\ Muhimbili University of Health and Allied Sciences \\ Workineh Shibeshi \\ Addis Ababa University \\ Aster Shewamare \\ Zewditu Memorial Hospital, ART Clinic \\ Ephrem Engidawork \\ Addis Ababa University
}

\section{Research}

Keywords: ART, Biomarkers, Cardiometabolic syndrome, HIV, Prevalence

Posted Date: March 15th, 2021

DOI: https://doi.org/10.21203/rs.3.rs-288131/v1

License: @ (i) This work is licensed under a Creative Commons Attribution 4.0 International License. Read Full License

Version of Record: A version of this preprint was published at Clinical Medicine Insights: Endocrinology and Diabetes on January 1st, 2022. See the published version at https://doi.org/10.1177/11795514221078029. 


\section{Abstract \\ Background}

Although marked improvements in life expectancy have been observed with the rapid expansion of Antiretroviral Therapy (ART), Cardiometabolic Syndrome (CMetS) is becoming a serious challenge for People Living with HIV/AIDS (PLWHA). The present study aimed in determining biomarkers and prevalence of CMetS in PLWHA.

\section{Methods}

A hospital-based, observational study was carried out between January 2019 \& February 2020 among HIV infected adults $(n=288)$. Binary logistic regression was used to estimate odds ratio (OR) and corresponding $95 \%$ confidence interval (Cl) for the association between the outcomes against the predictor variables.

\section{Results}

The current study revealed that the prevalence of CMetS was 28.5\% (82/288) using the National Cholesterol Education Program (NCEP)-2005 definition; and it was 43.5\% (126/288) using the International Diabetes Federation (IDF)-2005. Male gender was less likely to be associated with CMetS (OR $=.086$, C.I. $0.025-0.292, p<0.001)$ using the NCEP-2005 definition. Individuals with longer duration on ART have an increased odds of CMetS using both the NCEP-2005 (OR = 1.024, C.I. 1.005-1.043, $p=0.014)$ and the IDF-2005 (OR $=1.251$, C.I. 1.061-1.472, $p=0.007$ ) definitions. The age at which ART initiated yet have an impact on the outcomes of CMetS (NCEP-2005: OR= 1.27, C.I. 1.031-1.564, $p=0.025)$, indicating that individuals who started ART treatment at older age are more likely to have CMetS than their younger counterparts. The study further verified that, individuals with increased waist-grid (central adiposity) were more likely to have CMetS using both the NCEP-2005 (OR = 1.21, C.I. 1.029-1.418, $p=0.021)$ and the IDF-2005 (OR = 1.730 C.I. 1.454-2.058, $p<0.001)$ definitions. PLWHA with increased in DBP $(O R=1.164, C . I .1 .080-1.254, p<0.001)$, Triglyceride $(O R=1.027, C . I .0 .015-0.039, p<0.001)$, and low density lipoproteins $(\mathrm{OR}=1.075$, C.I. $0.020-0.134, \mathrm{p}=0.007)$ were more likely to have CMetS using the NCEP-2005 definition. PLWHA without comorbidity were less likely to have CMetS (NCEP-2005: OR $=0.086$, C.I. $0.025-0.292, p<0.001$ ).

\section{Conclusions}

The prevalence of CMetS in the study area was high. Risk factors associated with CMetS were waist circumference, gender, duration on ART; ART initiated age, waist-grid, and comorbidity. Biomarkers that were more likely contributed to the prevalence of CMetS include triglyceride, low density lipoproteins, and systolic blood pressure.

\section{Introduction}

People Living with HIV/AIDS (PLWHA) have been greatly benefited from the introduction of Antiretroviral Therapy (ART) in terms of quality of life, lengthen survival and psychological boost. However, medication and disease related problems have put the ART management in jeopardy [1-6]. Cardiometabolic Syndrome (CMetS) is a group of cardiac and metabolic derangement manifested with alteration on common biomarkers such as Impaired Glucose Tolerance (IGT), Insulin Resistance (IR), high serum Triglycerides (TG), low High-Density Lipoprotein Cholesterol (HDL-C) levels, hypertension, central adiposity, high Fasting Blood Sugar (FBS), and diseases involving the cardiac system [6-13].

Several definitions are being used to define CMetS including the Modified National Cholesterol Education Programme Adult Treatment Panel III (NCEP-ATP III)-2005, the International Diabetes Federation (IDF)-2005, the World Health Organization (WHO)-1998, the European Group for the Study of Insulin Resistance (EGIR)-1999, and the American Heart Association and the National Heart, Lung, and Blood Institute (AHA/ NHLBI) definitions [14-16]. Each definition has its distinctive reason and characterization of variable selection and except for the NCEP-ATP III definition, all the rest definition have an absolute requirement, whereas for the NCEP-2005 definition, there is no an absolute requirement. The five requirements are hypertension, hyperglycemia, dyslipidemia type 1 (measuring TGs) and dyslipidemia type 2 (measuring HDL-C), and waist-grid. Fulfilling three out of the five requirements are needed to define CMetS. Microalbuminuria measurement is an optional criterion for the WHO definition [14, 17-20]. The detail is available in annex-1 [14, 17-20]. 
Biomarkers or biological markers are objective indications of medical state that can be measured accurately and reproducibly [21]. Biomarkers provide a dynamic and powerful approach to understanding the spectrum of disease detection, progression, and monitoring [22]. Historically, biomarker were coined only to biological fluid, tissues, and chemicals that has been related and used to evaluate the disease conditions [23]. Nowadays, the term biomarker can also be used in various aspects outside biological samples as long as it is helpful in diagnosis, measuring, and monitoring disease conditions [21, 24]. HIV guidelines globally are focusing on HIV treatment and disease monitoring, giving less attention to emerging problems like CMetS, makes the standard of ART care imperfect [25-27]. Hence, the present study is aimed at determining the prevalence and biomarkers of CMetS among HIV infected adults on follow-up care.

\section{Methods}

\section{Study design, period and setting}

This was a hospital-based, observational study conducted during the period of 25/01/2019 to 25/02/2020 in HIV infected adults on follow-up care at Zewditu Memorial Hospital (ZMH), Addis Ababa, Ethiopia. The study was part of a large cohort study aimed at reporting the prevalence data. ZMH is the first hospital inaugurated as well as initiated ART service in Ethiopia, July 2003 [28, 29].

\section{Study population}

The study population was all PLWHA attending the ART follow-up care at ZMH. The target population was PLWHA with age $\geq 18$ years and willing to participate in the study.

\section{Sample size determination}

The following equation was used to calculate the sample size:-

Sample size $n=[D E F F * N p(1-p)] /[(d 2 / z 21-\alpha / 2 *(N-1)+p *(1-p)$

\begin{tabular}{|ll|}
\hline Population size (for finite population correction factor or FPC)(M): & 7674 \\
\hline Hypothesized \% frequency of outcome factor in the population $(p):$ & $26 \%+/-5[30]$ \\
\hline Confidence limits as \% of 100(absolute +/- \%) $(d):$ & $5 \%$ \\
\hline Design effect (for cluster surveys-DEFF): & 1 \\
\hline Confidence Level (\%) & $95 \%$ \\
\hline Sample Size & 286 \\
\hline $\begin{array}{l}\text { Where, N (7674) is the total HIV infected population registered for follow-up care and P is the prevalence for CMetS in HIV- infected } \\
\text { population obtained from published articles [30]. Considering 10\% contingency (lost to follow-up and defaulters), the final sample size } \\
\text { of the study became 314. }\end{array}$ \\
\hline
\end{tabular}

\section{Research Questions}

1. Do HIV infected persons in Ethiopia have a higher prevalence of CMetS?

2. What are the biomarkers associated with CMetS in this population?

3. What are the risk factors that predispose HIV-infected individuals for prevalence and progression of CMetS?

\section{Hypothesis}

The prevalence, biomarkers, and risk factors of CMetS among HIV infected persons in Ethiopia are not different from other similar studies.

\section{Sampling procedure and enrollment}

A systematic random sampling technique was used to recruit study participants. The sample interval $(\mathrm{K})$ was calculated using the

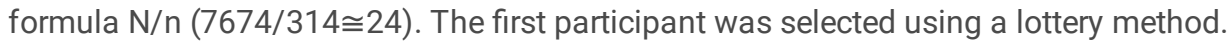

\section{Data collection}


Participants' information was collected in the form of interview and by tracking participants' charts. The questionnaire for a face-to-face interview was adapted from the structured questionnaire used by the WHO Stepwise approach to non-communicable disease risk factor surveillance (STEPS-2014) [31]. The questionnaire was composed of information related to socio-demographic characteristics and clinical characteristics. Data were collected by two trained data collectors who administered the questionnaire, performed anthropometric measurements, measured BP, and took blood samples for biomedical measurements. Anthropometric measurements including weight (in $\mathrm{Kg}$ ), Height (in meter), a derived Body Mass Index (BMI = weight in $\mathrm{kg} /$ height in $\mathrm{m}^{2}$ ), and Waist Grid Circumference/Abdominal Circumference (in inch) were taken.

\section{Data analysis}

Data was coded, double-entered, and analyzed using IBM SPSS Statistics 25. Descriptive and inferential statistics were used to present data. Binary logistic regression was used to estimate odds ratios (ORs) and corresponding $95 \%$ confidence intervals (Cls) for the association between the outcomes against the predictor variables. The mean of the repeated measures were used in cases of Pulse Rate (PR), Systolic Blood Pressure (SBP), and Diastolic Blood Pressure (DBP); whereas, for the other variables, the baseline data was used to determine the effect on the prevalence of CMetS. A 95\% C.I was used and the level of significance for statistical analysis was set at less than 0.05 .

Height and weight were measured by Type ZT-160 body-weight balance. Biomedical measurements such as PR (per minute), SBP $(\mathrm{mmHg}), \mathrm{DBP}(\mathrm{mmHg})$, and tests such as FBG $(\mathrm{mg} / \mathrm{dl})$, and Fasting Blood Cholesterol $(\mathrm{mg} / \mathrm{dl})$ were taken routinely during appointments (every six months). BP and PR were measured by Omron HEM 7203, a fully automatic digital blood pressure monitor (Omron Healthcare Co. Ltd., Kyoto, Japan). The devices were regularly calibrated for proper validation. Mercury sphygmomanometer was used for evaluating the accuracy of the devices. Three BP recordings were obtained from the right arm of the patients with an interval of 5 min and the mean of the three readings was used for the final result. All measurements were taken at baseline and then repeated after 6 months [32, 33].

An absolute CD4 count (AbsCD4) and per cent CD4 (\%CD4) were measured by BD FACSPresto ${ }^{\text {TM }}$ and BD FACSCalibur ${ }^{\text {TM }}$. Viral load was measured using the Abbott RealTime HIV-1 assay. Lipid profiles were measured using SIEMENS (Dimension EXL 200 Integrated Chemistry System) and the Omina Health (CS-T240 Auto-Chemistry Analyzer). The instruments analyze integrated clinical chemistry and immunoassay.

\section{Operational definition}

Biomarkers: are measurable indicators of some biological state or condition. Includes waist-grid, BMI, BP, Lipid profile, CD4 and VL measurements, and blood sugar measurements.

Risk factor or determinant: is a variable associated with an increased risk of disease or infection. Examples include: Age, Race/ethnicity, Gender, Some medical conditions, Use of certain medications, Poverty and crowding, certain occupations, Pregnancy.

\section{Results}

In this observational study, although 314 patients were initially enrolled, the final sample size used for analysis was $(n=288) H I V$ infected persons. Twenty-six patients were out of the analysis for various reasons: two were discontinued from follow-up due to change of addresses, four were due to critical illnesses (one due to HBS, three due to high BP), and the rest 10 were defaulter for unknown reasons (Fig. 1).

The demographic data illustrated that a slight preponderance of female (162,56.2\%), and nearly 1 in 2 were married, half 62 (21.5\%) were divorced, 1 in 3 were completed secondary high school of grade 9-12th education, and 1 in 4 were involved in small self-employed with employee business. Almost, $10 \%$ of the population was jobless and $4.5 \%$ were students. Majority 271 (94.1\%) were from Addis Ababa (see Table 1). 
Table 1

Socio-demographic information of the study participants: counts, percent of total and cumulative percent; Zewditu Memorial Hospital, June 2020, Addis Ababa, Ethiopia.

\begin{tabular}{|c|c|c|}
\hline Socio-demographic & Counts & $\%$ of Total \\
\hline \multicolumn{3}{|l|}{ Gender } \\
\hline Female & 162 & 56.3 \\
\hline Male & 126 & 43.8 \\
\hline \multicolumn{3}{|l|}{ Age } \\
\hline $20-34$ & 52 & 18.1 \\
\hline $35-39$ & 47 & 16.3 \\
\hline $40-44$ & 55 & 19.1 \\
\hline $45-49$ & 43 & 14.9 \\
\hline $50-54$ & 35 & 12.2 \\
\hline $55-59$ & 23 & 8.0 \\
\hline $60-64$ & 21 & 7.3 \\
\hline $65-69$ & 5 & 1.7 \\
\hline $70-74$ & 3 & 1.0 \\
\hline $75-79$ & 1 & .3 \\
\hline \multicolumn{3}{|l|}{ Civil status } \\
\hline Never married & 53 & 18.4 \\
\hline Married & 130 & 45.1 \\
\hline Divorced & 62 & 21.5 \\
\hline Widowed/r & 43 & 14.9 \\
\hline \multicolumn{3}{|l|}{ Educational status } \\
\hline No formal education & 35 & 12.2 \\
\hline Primary (1-6th grade) & 65 & 22.6 \\
\hline Secondary Junior (7-8th grades) & 27 & 9.4 \\
\hline Secondary High school (9-12th grades) & 96 & 33.3 \\
\hline College/university diploma & 47 & 16.3 \\
\hline College/ University Degree/master or above & 18 & 6.3 \\
\hline \multicolumn{3}{|l|}{ Occupational status $\#$} \\
\hline Higher-level professional & 1 & 0.3 \\
\hline Higher-level manager and entrepreneur & 1 & 0.3 \\
\hline Lower level professional & 27 & 9.4 \\
\hline Lower level manager & 3 & 1.0 \\
\hline Clerical routine non-manual worker & 11 & 3.8 \\
\hline Sales and service routine non-manual worker & 1 & 0.3 \\
\hline
\end{tabular}

\# Classification is based on ISEC (International Socio-Economic Classes) ${ }^{64}$. 


\begin{tabular}{|c|c|c|}
\hline Socio-demographic & Counts & $\%$ of Total \\
\hline Small self-employed with employee & 11 & 3.8 \\
\hline Small self-employed without employer & 71 & 24.7 \\
\hline Skilled manual worker & 21 & 7.3 \\
\hline Semi- and unskilled manual worker & 84 & 29.2 \\
\hline Agricultural laborer & 2 & 0.7 \\
\hline Retired & 13 & 4.5 \\
\hline Student & 13 & 4.5 \\
\hline Jobless & 29 & 10.1 \\
\hline \multicolumn{3}{|l|}{ Monthly income } \\
\hline$</=100$ USD & 215 & 74.7 \\
\hline$>100$ USD & 73 & 25.3 \\
\hline \multicolumn{3}{|l|}{ Address } \\
\hline Addis Ababa & 271 & 94.1 \\
\hline Gulelle & 4 & 1.4 \\
\hline Arada & 11 & 3.8 \\
\hline Kolfe & 7 & 2.4 \\
\hline Addis Ketema & 4 & 1.4 \\
\hline Nefas Silk Lafto & 38 & 13.2 \\
\hline Kirkos & 109 & 37.8 \\
\hline Lideta & 8 & 2.8 \\
\hline Yeka & 16 & 5.6 \\
\hline Bole & 16 & 5.6 \\
\hline Akaki-Kality & 58 & 20.1 \\
\hline Out of Addis Ababa & 17 & 5.9 \\
\hline
\end{tabular}

In view of the clinical background, most 131(44.8\%) were on stage III of the WHO classification and 276 (95.8\%) were on T1 classification. Most were on 1st line ART regimen 235 (81.6\%), whereas, 129 (44.8\%) were changed their initial regimen at least once (Table 2). Half $(139,48.3 \%)$ were on $1 j(T D F+3 T C+D T G)$ regimen, (Fig. 2). 
Table 2

Clinical information of the study participants: counts, percent of total and cumulative percent; Zewditu Memorial Hospital, June 2020, Addis Ababa, Ethiopia. $\mathrm{N}=288$.

\begin{tabular}{|c|c|c|}
\hline Clinical information & Counts & $\%$ of Total \\
\hline \multicolumn{3}{|l|}{ The WHO clinical staging } \\
\hline I & 40 & 13.9 \\
\hline II & 68 & 23.6 \\
\hline III & 131 & 45.5 \\
\hline IV & 49 & 17.0 \\
\hline \multicolumn{3}{|l|}{ Treatment staging } \\
\hline $\mathrm{T} 1$ & 276 & 95.8 \\
\hline $\mathrm{T} 2$ & 1 & .3 \\
\hline T3 & 7 & 2.4 \\
\hline T4 & 4 & 1.4 \\
\hline \multicolumn{3}{|l|}{ ART regimen } \\
\hline First line & 235 & 81.6 \\
\hline Second line & 51 & 17.7 \\
\hline Third line & 2 & .7 \\
\hline \multicolumn{3}{|l|}{ Frequency of ART regimen change } \\
\hline No change from the baseline & 59 & 20.5 \\
\hline changed once & 129 & 44.8 \\
\hline changed twice & 80 & 27.8 \\
\hline changed thrice & 17 & 5.9 \\
\hline changed four times & 3 & 1.0 \\
\hline \multicolumn{3}{|l|}{ ART regimen } \\
\hline 2NRTIS + 1INSTI & 140 & 48.6 \\
\hline 2NNRTIs + 1NNRTI & 96 & 33.3 \\
\hline 2NRTIs + 1PI & 50 & 17.4 \\
\hline 1NRTI + 1NNRTI + 1INSTI + 1PI & 2 & 0.7 \\
\hline \multicolumn{3}{|l|}{ CD4 count (cells/mm3) } \\
\hline$<50$ & 5 & 1.7 \\
\hline $51-200$ & 36 & 12.5 \\
\hline $201-500$ & 142 & 49.3 \\
\hline$>500$ & 105 & 36.5 \\
\hline \multicolumn{3}{|l|}{ VL (copies/mL) } \\
\hline LDL ** $(<150)$ & 219 & 76.0 \\
\hline Low viral load (151-1000) & 11 & 3.8 \\
\hline Intermediate viral load $(1000-10,000)$ & 25 & 8.7 \\
\hline${ }^{\star \star}$ LDL $=$ Low Detection Level & & \\
\hline
\end{tabular}




\begin{tabular}{|lll|}
\hline Clinical information & Counts & $\%$ of Total \\
\hline High viral load $(>10,000)$ & 33 & 11.5 \\
\hline Cardiometabolic syndrome & & \\
\hline NCEP-2005 & 82 & 28.5 \\
\hline Cardiometabolic syndrome & & \\
\hline IDF-2005 & 126 & 43 \\
\hline$* \star$ LDL $=$ Low Detection Level & & \\
\hline
\end{tabular}

The overall mean age of the population was 43.51 with standard deviation (SD) of \pm 11.273 . That is about, $40.7 \pm 10.5$ for women, and $47.1 \pm 11.4$ for men. The overall mean age at the time of HIV confirmation was $32.7 \pm 11.1$, where, $30.2 \pm 10.4$ were women, and $35.9 \pm$ 11.4 were men. The details of the characteristics of subjects are shown in Table 3. 
Table 3

Characteristics of subjects $(\mathrm{N}=288)$ enrolled in the study, overall and by gender, Zewditu Memorial Hospital, June 2020, Addis Ababa, Ethiopia.

\begin{tabular}{|c|c|c|c|c|c|c|c|c|c|c|c|c|}
\hline \multirow{2}{*}{$\begin{array}{l}\text { Characteristics } \\
\text { Gender }\end{array}$} & \multicolumn{3}{|c|}{ Minimum } & \multicolumn{3}{|c|}{ Maximum } & \multicolumn{3}{|c|}{ Median } & \multicolumn{3}{|c|}{ Mean \pm Std. Deviation } \\
\hline & $\begin{array}{l}\text { Female } \\
(\mathrm{F})\end{array}$ & $\begin{array}{l}\text { Male } \\
\text { (M) }\end{array}$ & $F+M$ & $\mathrm{~F}$ & $\mathrm{M}$ & $F+M$ & $\mathrm{~F}$ & $\mathrm{M}$ & $\begin{array}{l}\mathrm{F}+ \\
\mathrm{M}\end{array}$ & $F$ & $\mathrm{M}$ & $\mathrm{F}+\mathrm{M}$ \\
\hline Age (year) & 20.0 & 20.0 & 20.0 & 69.0 & 77.0 & 77.0 & 40.0 & 47.0 & 43.0 & $\begin{array}{l}40.7 \pm \\
10.5\end{array}$ & $\begin{array}{l}47.1 \pm \\
11.4\end{array}$ & $\begin{array}{l}43.5 \pm \\
11.3\end{array}$ \\
\hline $\begin{array}{l}\text { Monthly } \\
\text { income (in } \\
\text { birr) }\end{array}$ & 0.00 & 0.00 & 0.00 & 10000 & 40000 & 40000 & 2000 & 3000 & 2000 & $\begin{array}{l}2177 \\
\pm 2029\end{array}$ & $\begin{array}{l}4009 \\
\pm 5597\end{array}$ & $\begin{array}{l}2979 \\
\pm 4097\end{array}$ \\
\hline $\begin{array}{l}\text { Age@ confirm } \\
\text { (year) }\end{array}$ & 7.00 & 7.00 & 7.00 & 63.0 & 65.0 & 65 & 29.0 & 36.0 & 32 & $\begin{array}{l}30.2 \pm \\
10.4\end{array}$ & $\begin{array}{l}35.9 \pm \\
11.2\end{array}$ & $\begin{array}{l}32.7 \pm \\
11.1\end{array}$ \\
\hline $\begin{array}{l}\text { Age@ start } \\
\text { (year) }\end{array}$ & 7.00 & 8.00 & 7.00 & 67.0 & 65.0 & 67.0 & 30.0 & 37.0 & 33.0 & $\begin{array}{l}31.1 \pm \\
10.6\end{array}$ & $\begin{array}{l}36.8 \pm \\
11.2\end{array}$ & $\begin{array}{l}33.6 \pm \\
11.2\end{array}$ \\
\hline $\begin{array}{l}\text { Total years on } \\
\text { ART }\end{array}$ & 0.460 & 0.633 & 0.460 & 22.2 & 18.5 & 22.2 & 9.55 & 12.0 & 11.2 & $\begin{array}{l}9.51 \pm \\
4.66\end{array}$ & $\begin{array}{l}10.2 \pm \\
4.68\end{array}$ & $\begin{array}{l}9.81 \pm \\
4.67\end{array}$ \\
\hline $\begin{array}{l}\text { Current weight } \\
(\mathrm{Kg})\end{array}$ & 33.0 & 39.0 & 33.0 & 93.0 & 120 & 120 & 57.0 & 67.5 & 60.0 & $\begin{array}{l}58.5 \pm \\
11.1\end{array}$ & $\begin{array}{l}68.0 \pm \\
13.0\end{array}$ & $\begin{array}{l}62.7 \pm \\
12.8\end{array}$ \\
\hline $\begin{array}{l}\text { Current height } \\
\text { (in meter) }\end{array}$ & 1.44 & 1.50 & 1.44 & 1.71 & 1.91 & 1.91 & 1.57 & 1.70 & 1.63 & $\begin{array}{l}1.58 \pm \\
0.0594\end{array}$ & $\begin{array}{l}1.70 \pm \\
0.0700\end{array}$ & $\begin{array}{l}1.63 \pm \\
0.087\end{array}$ \\
\hline BMI $\left(\mathrm{kg}^{2} / \mathrm{m}^{2}\right)$ & 14.5 & 15.1 & 14.5 & 39.2 & 35.1 & 39.2 & 23.1 & 23.2 & 23.1 & $\begin{array}{l}23.6 \pm \\
4.26\end{array}$ & $\begin{array}{l}23.6 \pm \\
4.09\end{array}$ & $\begin{array}{l}23.6 \pm \\
4.18\end{array}$ \\
\hline $\begin{array}{l}\text { Abd. Circ. } \\
\text { (inch) }\end{array}$ & 24.0 & 25.0 & 24.0 & 49 & 49 & 49.0 & 32.0 & 35.0 & 33.5 & $\begin{array}{l}33.4 \pm \\
4.36\end{array}$ & $\begin{array}{l}35.6 \pm \\
4.47\end{array}$ & $\begin{array}{l}34.3 \pm \\
4.53\end{array}$ \\
\hline $\begin{array}{l}\text { CD4 } \\
\left(\text { cells } / \mathrm{mm}^{3}\right)\end{array}$ & 74.0 & 6.00 & 6.00 & 1294 & 1406 & 1406 & 431 & 402 & 410 & $\begin{array}{l}468 \pm \\
266\end{array}$ & $\begin{array}{l}427 \pm \\
244\end{array}$ & $\begin{array}{l}450 \pm \\
257\end{array}$ \\
\hline $\begin{array}{l}\text { VL } \\
\text { (copies/mL) }\end{array}$ & 0.00 & 0.00 & 0.00 & 466201 & 466900 & 466900 & 0.00 & 0.00 & 0.00 & $\begin{array}{l}11964 \\
\pm \\
56320\end{array}$ & $\begin{array}{l}1301 \\
\pm \\
55092\end{array}$ & $\begin{array}{l}12422 \\
\pm \\
55692\end{array}$ \\
\hline $\begin{array}{l}\text { PR (heart- } \\
\text { beat/minute) }\end{array}$ & 47.7 & 60.7 & 47.7 & 123 & 116 & 123 & 82.7 & 79.7 & 82.0 & $\begin{array}{l}83.9 \pm \\
11.5\end{array}$ & $\begin{array}{l}79.9 \pm \\
10.1\end{array}$ & $\begin{array}{l}82.1 \pm \\
11.1\end{array}$ \\
\hline $\mathrm{SBP}(\mathrm{mmHg})$ & 89.3 & 91.3 & 89.3 & 193 & 213 & 213 & 121 & 128 & 125 & $\begin{array}{l}126 \pm \\
21.3\end{array}$ & $\begin{array}{l}131 \pm \\
22.2\end{array}$ & $\begin{array}{l}128 \pm \\
21.9\end{array}$ \\
\hline DBP (mmHg) & 54.7 & 61.7 & 54.7 & 117 & 118 & 118 & 80.3 & 83.0 & 81.3 & $\begin{array}{l}81.7 \pm \\
11.2\end{array}$ & $\begin{array}{l}84.5 \pm \\
12.5\end{array}$ & $\begin{array}{l}82.9 \pm \\
11.8\end{array}$ \\
\hline $\mathrm{TC}(\mathrm{mg} / \mathrm{dL})$ & 78.0 & 100 & 78.0 & 354 & 492 & 492 & 184 & 185 & 184 & $\begin{array}{l}185 \pm \\
48.5\end{array}$ & $\begin{array}{l}191 \pm \\
55.2\end{array}$ & $\begin{array}{l}187 \pm \\
51.6\end{array}$ \\
\hline $\begin{array}{l}\text { LDL-C } \\
(\mathrm{mg} / \mathrm{dL})\end{array}$ & 21.0 & 33.0 & 21.0 & 306 & 615 & 615 & 117 & 110 & 117 & $\begin{array}{l}117 \pm \\
49.0\end{array}$ & $\begin{array}{l}121 \pm \\
67.4\end{array}$ & $\begin{array}{l}118 \pm \\
57.7\end{array}$ \\
\hline TGs (mg/dL) & 49.0 & 30.0 & 30.0 & 466 & 491 & 491 & 114 & 153 & 133 & $\begin{array}{l}139 \pm \\
74.2\end{array}$ & $\begin{array}{l}177 \pm \\
98.0\end{array}$ & $\begin{array}{l}155 \pm \\
87.3\end{array}$ \\
\hline $\begin{array}{l}\text { HDL-C } \\
(\mathrm{mg} / \mathrm{dL})\end{array}$ & 21.0 & 21.0 & 21.0 & 69.8 & 67.0 & 69.8 & 46.0 & 46.0 & 46.0 & $\begin{array}{l}46.5 \pm \\
7.39\end{array}$ & $\begin{array}{l}46.2 \pm \\
8.33\end{array}$ & $\begin{array}{l}46.4 \pm \\
7.80\end{array}$ \\
\hline $\begin{array}{l}\text { VLDL-C } \\
(\mathrm{mg} / \mathrm{dL})\end{array}$ & 14.0 & 7.00 & 7.00 & 229 & 197 & 229 & 22.0 & 26.0 & 24.0 & $\begin{array}{l}26.6 \pm \\
19.4\end{array}$ & $\begin{array}{l}30.2 \pm \\
19.1\end{array}$ & $\begin{array}{l}28.2 \pm \\
19.3\end{array}$ \\
\hline $\begin{array}{l}\text { Non-HDL-C } \\
(\mathrm{mg} / \mathrm{dL})\end{array}$ & 40.0 & 39.0 & 39.0 & 313 & 451 & 451 & 140 & 140 & 140 & $\begin{array}{l}138 \pm \\
49.8\end{array}$ & $\begin{array}{l}144 \pm \\
57.4\end{array}$ & $\begin{array}{l}141 \pm \\
53.2\end{array}$ \\
\hline $\begin{array}{l}\text { Fasting Blood } \\
\text { glucose } \\
(\mathrm{mg} / \mathrm{dL})\end{array}$ & 60.0 & 45.0 & 45 & 140 & 319 & 319 & 90.0 & 98.0 & 94.0 & $\begin{array}{l}94.6 \pm \\
15.8\end{array}$ & $\begin{array}{l}101 \pm \\
30.5\end{array}$ & $\begin{array}{l}97.3 \pm \\
23.6\end{array}$ \\
\hline
\end{tabular}




\begin{tabular}{|l|l|}
\hline Characteristics Minimum Maximum & Median \\
\hline Age@ confirm = Age at Confirmation of HIV; Age@ $@$ start = Age during initiation of ART; Abd. Circ.=abdominal circumference; VL = Viral \\
Load; PR=; SBP = Systolic Blood Pressure; DBP = Diastolic Blood Pressure; TC = Total Cholesterol; LDL-C = Low-Density Lipoprotein; \\
TGs= Triglyceride, HDL-C = High Density Lipoprotein; VLDL-C = Very- Low- Density Lipoprotein. \\
Current Birr to USD conversion rate is 0.0251.
\end{tabular}

The shortest duration that the participant had on ART follow-up care was 0.46 years, whereas the longest duration was 22.2 years. The overall total years on HAART was $9.81+4.67$ (mean + SD). The youngest age at the time of HIV confirmation \& HAART initiation was 7 years and the oldest age among participant for HIV confirmation was 63 for women and 65 for men, and for ART initiation, 67 for women and 65 for men (Table 3).

The prevalence of CMetS using the NCEP-2005 definition was 28.5\% (82/288); and it was 43.5\% (126/288) using the IDF definition (Table 2).

In order to determine the impact of biomarkers, risk factors, related sociodemographic variables on the prevalence of CMetS, two separate binary logistic analyses were performed for each CMetS_NCEP and CMetS_IDF definitions. Hence, twenty three predictor variables were entered to see their impact on the outcome of CMetS using the NCEP-2005 definition: Age, Gender, Family_history, Current_DX, Waist_circumference in inch, SBP in mmHg, DBP in $\mathrm{mmHg}$, Total cholesterol in $\mathrm{mg} / \mathrm{dL}$, TG in mg/dL, LDL_C in mg/dL, Current_Tobacco_use (regular with intermittent use vs no use at all), Current_Alcohol_use (regular with intermittent use vs no use at all), Current_Coffee_use (regular with intermittent use vs no use at all), BMI in kg/m2, Duration_with_ART in year, nadir CD4 count in Cells $/ \mathrm{mm}^{3}$, weight gained since HAART initiation in $\mathrm{Kg}$, Frequency of ART Change (changed once or more time vs no change at all), Blood Group (A, B, AB, O), Rh Factor (Rh + vs Rh-), HAART_start_age in year.

The full model containing all the predictors was statistically significant $X^{2}(23 ; 288)=191.443$, indicating that the model was able to distinguish b/n those with and without CMetS. The total model explained b/n 48.6\% (Cox and Snell R squared) and 69.6\% (Nagelkereke R Square) of the variance in CMetS and correctly classified $89.6 \%$ of the cases. Eleven of the predictor variables (Age, gender, waist circumference, comorbidity, DBP, TG, LDL-C, duration with ART, blood group, and HAART_start_age) made a statistically significant contribution to the model (see Table 4). The strongest predictors were waist circumference, DBP and ART start age, which had an odds ratio of $1.21,1.16$, and 1.27 respectively. Demonstrating for each inch increment on waist circumference the odds of CMetS increases by 1.21 factors ( $21 \%$ more likely to develop CMetS), and similarly the odds of CMetS increases by $1.16(16 \%)$ for each mmHg increment in DBP and also CMetS increases by $1.27(27 \%)$ for each year increment in ART start age. 
Table 4

The effect of the cardiometabolic predictors on the outcome of cardiometabolic syndrome using the National Cholesterol Education Program Adult Treatment Panel III (NCEP_ATP III-2005) definition. Data extracted from the cohort study carried out on HIV-infected persons on follow care of Zewditu Memorial hospital, Addis Ababa, Ethiopia, 2020.

\begin{tabular}{|c|c|c|c|c|c|c|c|}
\hline \multirow[t]{2}{*}{ Variables in the Equation } & \multirow[t]{2}{*}{ Odds } & \multirow[t]{2}{*}{ S.E. } & \multirow[t]{2}{*}{ Odds Ratio } & \multicolumn{2}{|c|}{ 95\% C.I.for OR } & \multirow[t]{2}{*}{ Wald Statistics } & \multirow[t]{2}{*}{$P$} \\
\hline & & & & Lower & Upper & & \\
\hline Age & -.240 & .111 & .786 & .633 & .977 & 4.716 & $.030 *$ \\
\hline Gender (male) & -2.455 & .625 & .086 & .025 & .292 & 15.416 & $.000 * \star$ \\
\hline Family history (Yes) & -.141 & .566 & .869 & .286 & 2.635 & .062 & .804 \\
\hline Co-morbidity (No) & -3.126 & 1.196 & .044 & .004 & .457 & 6.835 & $.009 *$ \\
\hline Waist circumference (inch) & .189 & .082 & 1.208 & 1.029 & 1.418 & 5.361 & $.021^{\star}$ \\
\hline $\mathrm{SBP}(\mathrm{mmHg})$ & -.014 & .018 & .986 & .951 & 1.022 & .579 & .447 \\
\hline $\mathrm{DBP}(\mathrm{mmHg})$ & .152 & .038 & 1.164 & 1.080 & 1.254 & 15.912 & $.000 * *$ \\
\hline TG (mg/dL) & .027 & .006 & 1.027 & 1.015 & 1.039 & 19.614 & $.000 * *$ \\
\hline LDL_C (mg/dL) & .073 & .027 & 1.075 & 1.020 & 1.134 & 7.193 & $.007 *$ \\
\hline Current_Tobacco_use (yes) & -1.984 & 1.120 & .137 & .015 & 1.234 & 3.141 & .076 \\
\hline Current_Alcohol_use (Yes) & -.964 & .865 & .381 & .070 & 2.080 & 1.240 & .265 \\
\hline Current_Coffee_use (Yes) & .471 & .453 & 1.602 & .659 & 3.893 & 1.082 & .298 \\
\hline $\mathrm{BMI}\left(\mathrm{kg} / \mathrm{m}^{2}\right)$ & .110 & .092 & 1.116 & .932 & 1.337 & 1.425 & .233 \\
\hline Duration on ART (year) & .024 & .010 & 1.024 & 1.005 & 1.043 & 6.030 & $.014^{\star}$ \\
\hline nadir cd4 count (cells $/ \mathrm{mm}^{3}$ ) & -.001 & .002 & .999 & .994 & 1.004 & .211 & .646 \\
\hline weight gained since HAART initiation $(\mathrm{Kg})$ & .039 & .035 & 1.040 & .971 & 1.114 & 1.238 & .266 \\
\hline Frequency of ART change (Yes) & -.623 & .587 & .536 & .170 & 1.695 & 1.126 & .289 \\
\hline Blood group & & & & & & 12.128 & $.007^{\star}$ \\
\hline Blood group(A) & -1.541 & .589 & .214 & .068 & .679 & 6.848 & $.009 *$ \\
\hline Blood group(B) & .250 & .599 & 1.284 & .397 & 4.153 & .174 & .677 \\
\hline Blood group(AB) & .929 & .704 & 2.533 & .638 & 10.062 & 1.743 & .187 \\
\hline Rh Factor(+ ve) & 1.030 & .709 & 2.800 & .697 & 11.242 & 2.108 & .147 \\
\hline ART_start_age (year) & .239 & .106 & 1.270 & 1.031 & 1.564 & 5.044 & $.025^{\star}$ \\
\hline \multicolumn{8}{|l|}{ *Significant values ** $P<0.01$} \\
\hline \multicolumn{8}{|c|}{$\begin{array}{l}\text { a. Variable(s) entered on step 1: Age, Gender, Family history, Current_DX, Waist circumference in inch, AV_SBP, AV_DBP, AV_Total } \\
\text { cholestrol, AV_TG, AV_LDL_C, Current_Tobacco_use, Current_Alcohol_use, Current_Coffee_use, BMI = kg/m2, Duration with ART, nadir } \\
\text { cd4 count, wait gain since HART initiation, Frequency of ART change, Blood group, Rh Factor, HAART_start_AGE. }\end{array}$} \\
\hline \multicolumn{8}{|c|}{$\begin{array}{l}\text { b. Family history: about the confirmation of Cardiometabolic disease by physicians such as hypertension, Diabetes Mellitus, Heart } \\
\text { disease, dyslipidemia. }\end{array}$} \\
\hline
\end{tabular}

As age increases the odds of acquiring CMetS decreases by $21.4 \%(P=0.03)$. Furthermore, male gender was less likely to acquire CMetS than female by $91.4 \%(p<0.001)$. For an additional one $\mathrm{mg} / \mathrm{dL}$ increase in TG or LDL, the odds of CMetS was increased by $1.5 \%$ and $2 \%$ respectively. Increased in SBP however not determinant for CMetS and in each $\mathrm{mmHg}$ increase in SBP $1.4 \%$ less likely develop CMetS. For each year increase in duration with ART, the odds of CMetS increases by $2.4 \%$. This is also true for the case of ART start age and as the patient get older to start ART, the odds of CMetS is increased by a factor of 2.23 per year. Blood group ' $\mathrm{A}$ ' is less likely to be associated with CMetS while ' $O$ ' is highly associated. 
Similarly, coded and hypothesized variables were also checked for their impact on the CMetS prevalence using the IDF-2005 definition.

The full model containing all predictors had a statistically significant association, $X^{2}(23 ; 288)=202.268$, representing that the model was able to distinguish b/n those with and without CMetS. The total model explained b/n 50.5\% (Cox and Snell R square) and 67.6\% (Nagelkereke R Square) of the variance in CMetS and correctly classified $85.1 \%$ of the cases. Only five of the predictor variables (Waist Circumference, TG, LDL-C, duration with ART, and Frequency of ART change) had a statistically significant contribution to the model (see Table 5). The strongest predictors were waist circumference and duration with ART. Indicating that for each additional inch in waist circumference, the odds of CMetS is increases by a factor of 1.73 (73\%) and in each year increment in duration with ART, the odds of CMetS increases by $6.7 \%$. 
Table 5

The effect of the cardiometabolic predictors on the outcome of cardiometabolic syndrome using the International Diabetes Federation (IDF-2005) definition. Data extracted from a cohort study carried out on HIV-infected persons on follow care of Zewditu Memorial hospital, Addis Ababa, Ethiopia, 2020.

\begin{tabular}{|c|c|c|c|c|c|c|c|}
\hline \multirow[t]{2}{*}{ Variables in the Equation } & \multirow[t]{2}{*}{ Odds } & \multirow[t]{2}{*}{ S.E. } & \multirow[t]{2}{*}{ Odds Ratio } & \multicolumn{2}{|c|}{ 95\% C.I.for OR } & \multirow[t]{2}{*}{ Wald Statistics } & \multirow[t]{2}{*}{$P$} \\
\hline & & & & Lower & Upper & & \\
\hline Age & -.033 & .080 & .968 & .827 & 1.133 & .166 & .684 \\
\hline \multicolumn{8}{|l|}{ Gender } \\
\hline Family history (Yes) & -.382 & .542 & .682 & .236 & 1.975 & .497 & .481 \\
\hline Co-morbidity (No) & .568 & .937 & 1.764 & .281 & 11.076 & .367 & .545 \\
\hline Waist circumference (inch) & .548 & .089 & 1.730 & 1.454 & 2.058 & 38.121 & $.000 * \star$ \\
\hline $\mathrm{SBP}(\mathrm{mmHg})$ & .006 & .016 & 1.006 & .975 & 1.039 & .157 & .692 \\
\hline $\mathrm{DBP}(\mathrm{mmHg})$ & .001 & .029 & 1.001 & .946 & 1.060 & .003 & .959 \\
\hline TG (mg/dL) & .015 & .005 & 1.015 & 1.004 & 1.025 & 7.447 & $.006^{*}$ \\
\hline LDL_C (mg/dL) & .062 & .026 & 1.064 & 1.012 & 1.119 & 5.801 & $.016^{*}$ \\
\hline Current_Tobacco_use (yes) & .019 & .011 & 1.019 & .997 & 1.042 & 2.799 & .094 \\
\hline Current_Alcohol_use (Yes) & -1.688 & 1.059 & .185 & .023 & 1.473 & 2.541 & .111 \\
\hline Current_Coffee_use (Yes) & -.857 & .737 & .424 & .100 & 1.800 & 1.351 & .245 \\
\hline $\mathrm{BMI}\left(\mathrm{kg} / \mathrm{m}^{2}\right)$ & .351 & .410 & 1.421 & .636 & 3.175 & .734 & .392 \\
\hline Duration with ART (year) & .224 & .083 & 1.251 & 1.064 & 1.472 & 7.324 & $.007 *$ \\
\hline nadir cd4 count (cells $/ \mathrm{mm}^{3}$ ) & .000 & .007 & 1.000 & .987 & 1.014 & .000 & .997 \\
\hline weight gained since HAART initiation $(\mathrm{Kg})$ & .003 & .002 & 1.003 & .999 & 1.007 & 2.766 & .096 \\
\hline Frequency of ART change (Yes) & -.077 & .029 & .926 & .876 & .980 & 7.187 & $.007 *$ \\
\hline Blood group & .677 & .482 & 1.967 & .766 & 5.055 & 1.975 & .160 \\
\hline Blood group(A) & & & & & & 2.720 & .437 \\
\hline Blood group(B) & .753 & .474 & 2.124 & .838 & 5.382 & 2.520 & .112 \\
\hline Blood group(AB) & .424 & .529 & 1.528 & .542 & 4.305 & .643 & .423 \\
\hline Rh Factor(+ ve) & .084 & .746 & 1.088 & .252 & 4.695 & .013 & .910 \\
\hline HAART_start_age (year) & .802 & .693 & 2.229 & .573 & 8.664 & 1.339 & .247 \\
\hline \multicolumn{8}{|l|}{ *Significant values ** $P<0.01$} \\
\hline \multicolumn{8}{|c|}{$\begin{array}{l}\text { d. Variable(s) entered on step 1: Age, Family history, Current_DX, Waist circumference in inch, AV_SBP, AV_DBP, AV_Total cholestrol, } \\
\text { AV_TG, AV_LDL_C, Current_Tobacco_use, Current_Alcohol_use, Current_Coffee_use, BMI = kg/m2, Duration with ART, nadir cd4 count, } \\
\text { wait gain since HAART initiation, Frequency of ART change, Blood group, Rh Factor, HAART_start_AGE. }\end{array}$} \\
\hline \multicolumn{8}{|c|}{$\begin{array}{l}\text { e. Family history: about the confirmation of Cardiometabolic disease by physicians such as hypertension, Diabetes Mellitus, Heart } \\
\text { disease, dyslipidemia. }\end{array}$} \\
\hline
\end{tabular}

Whereas for waist circumference, for each inch increment there is a 1.73 times more chance of developing CMetS. Individuals who have changed their initial ART regimens at least once have less likely to develop CMetS (7.4\% less likely), and TG and LDL have also contributed $1.5 \%$ and $6.4 \%$ odds of CMetS respectively.

\section{Discussion}


Antiretroviral treatment has modified the HIV progression, lengthened survival, and improved quality of life among PLWHA [34, 35]. However this overwhelming benefit is not without limitations. In recent time, PLWHA are at increased risk of cardiometabolic (CMet) derangements [36-39].

The prevalence data of CMetS among HIV-infected persons varies from country to country. In our study the prevalence using the NCEP2005 definition was $28.5 \%$ (82/288); and it was 43.5\% (126/288) using the IDF definition. A compiled report of studies showed that prevalence of CMetS in HIV infected population was $20.6 \%$ using NCEP-2005, and $31.3 \%$ using the IDF-2005 [40]. By considering this report as a reference, the chi-square goodness of fit test $(2 X 2)$ result indicated that the prevalence in our study was significantly higher by using both the NCEP-2005 ( $\left.X^{2}=121.94, d f=1, p<0.001\right)$, and the IDF-2005 models $\left(X^{2}=32.99, d f=1, p<0.001\right)$ [40]. However, in comparison to individual study reports, our prevalence falls within the range of previously done studies of [41-44], and in other aspect, it is a bit higher than the Polish [45], SHIVA (France) [46], Australian [47], South Korean [48], the Ethiopian [8, 44, 49], and the global meta analysis [50] studies; while it is lower than those reported from Nigeria [51], and China [52]. This variability could be due to the difference in study design, sample size, population genetics, study duration, duration with HIV and ART, treatment regimen or switch of therapy, and socio-demographic differences.

Several studies reported that sex, age, weight, BMI, sedentary lifestyle, central obesity, and cigarette smoking had an impact on the prevalence, pathogenesis, and progression of CMetS ${ }^{2,18-21}$. The European AIDS Clinical Society (EACS) guidelines has strongly addressed that the risk of contracting CMetS is age related [49]. In our study, male gender is less likely to be associated with CMetS (OR $=.086$, C.I. $0.025-0.292, \mathrm{p}<0.001)$ using the NCEP-2005 model; and this is in agreement with the studies reported from Latin America [53, 54] and Ethiopia [55]. However, a study from South Africa reported that the prevalence was higher in males [56].

Individuals with longer duration on ART had an increased odds of CMetS (NCEP-2005: OR $=1.024$, C.I. $1.005-1.043, p=0.014)$ and (IDF2005: $O R=1.251, C . I .1 .061-1.472, p=0.007)$. This result is similar to the study reported from Malawi where the duration of ART $>3$ years was associated with CMetS. Moreover, a Kenyan study reported that a step-up increment in the duration of ART is significantly related to an increased in CMetS [57]. The long-term exposure to ART could transform into considerable persistent metabolic risk [58-60]. However, the increase in age alone is not a determinant for the prevalence of CMetS. As per the result of our study, age increment was less likely to be associated with CMetS $(\mathrm{OR}=.786, \mathrm{C} . \mathrm{I} .0 .633-0.977, \mathrm{p}=0.03)$. This could be due to the fact that CMetS is more prevalent in age between 45 to 65 years [61] and the prevalence of CMetS could then decline thereafter [54]. Age 65 and above have fewer CMetS episodes than their younger counterparts for a number of reasons [62], one could be people who developed CMetS earlier than 60 could die prematurely before reaching 65 and above, the other is it could be due to the fact that diet has an impact on CMetS where elders are usual undernourished [63]. A life expectancy of many nations in the world is also under 65 and can affect the outcome [64]. Moreover, the incidence of CMetS is independent of patient age, and it is rather higher in patients who have other risk factors such as smoking [37].

The age at which ART initiated yet had an impact on the outcomes of CMetS (NCEP-2005: OR = 1.27, C.I. 1.031-1.564, p = 0.025), indicates individuals who started ART treatment at older age are more likely to have CMetS than their younger counterparts. Contrary to this finding, the CMet to childhood association was symmetrical in non-HIV infected population [65].

Our study further identified that, an increase in waist-grid (central adiposity) was associated with the prevalence of CMetS using both the NCEP-2005 (OR $=1.21$, C.I. $1.029-1.418, p=0.021)$ and the IDF-2005 models $(O R=1.730$ C.I. $1.454-2.058, p<0.001)$. This finding is welldocumented in a number of other studies [39, 48, 66-69]. Additionally, central adeposity can also contribute to CMetS due to its positive correlation with the DBP as described elsewhere $[70,71]$.

Individuals with increased TG were more likely to have CMetS using both the NCEP-2005 (OR $=1.027, \mathrm{C} . \mathrm{I} .0 .015-0.039, \mathrm{p}<0.001)$ and the IDF-2005 models ( $O R=1.015$ C.I. $0.004-0.025, p<0.001)$; similarly individuals with increased LDL were more likely to have CMetS using the NCEP-2005 (OR $=1.075$, C.I. $0.020-0.134, p=0.007$ ) as well as the IDF-2005 models $(O R=1.064, C . I .0 .012-0.119, p=0.016)$. These result are in harmony with a number of other publications $[37,69,72,73]$. Studies also indicated that if lipid abnormalities are not treated as aggressively as individuals living without HIV, and this can pose severe management crisis and premature fatalities in PLWHA [74].

Concerning the pattern of ART medication, $48.6 \%$ of the participants in our study were on 2 NRTIs +1 INSTI regimen, $33.3 \%$ on 2 NRTIs + $1 \mathrm{NNRTI}, 17.4 \%$ on $2 \mathrm{NRTIS}+1 \mathrm{PI}$, and $0.7 \%$ on $1 \mathrm{NRTI}+1 \mathrm{NNRTI}+1 \mathrm{INSTI}+1 \mathrm{PI}$ regimen. This regimen pattern was similar to a number of other studies [30, 47, 53]. Besides, the role of ART in the development and progression of CMetS has been elucidated in several other studies, but no significant result was obtained in our study $[68,75]$. On the other hand, individuals who had changed their baseline ART regimen had less likely to have CMetS (IDF-2005: OR $=0.926, C . I .0 .876-0.980, p=0.007$ ). This could be the newer ART medications have relatively fewer $\mathrm{CMet}$ effect than the orders [76], though this is still a point of debate among the scientific community. Several studies

Page $14 / 21$ 
reported that ART regimens such as PIs and NNRTIs are considered as a significant contributor to the development and progression of CMetS in HIV infected population [77-81].

PLWHA without comorbidity were less likely to have CMetS (NCEP-2005: OR $=0.086$, C.I. $0.025-0.292, p<0.001$ ). Among the comorbidities notably, Type 2 diabetes mellitus (T2DM), heart failure (HF), dyslipidemia and high BP were known to have a direct impact on the emergence of CMetS $[82,83]$.

\section{Conclusions}

The prevalence of CMetS using the NCEP-2005 definition was 28.5\% (82/288); and it was 43.5\% (126/288) using the IDF definition. Risk factors associated with CMetS were waist circumference, gender, duration on ART; ART initiated age, waist-grid, and comorbidity. Biomarkers more likely contributed to the prevalence of CMetS were triglyceride, low density lipoproteins, and systolic blood pressure.

\section{Recommendations}

A multicentre, multinational study with larger sample size and follow-up period is recommended. Strategies of lowering hypertriglyceridemia and boosting the HDL cholesterols are beneficial for HIV infected individuals and this can be initially guided by lifestyle modifications and appropriate selection of ART medications

\section{Abbreviations And Acronyms}

ART: Antiretroviral Therapy; ATP III: Adult Treatment Panel III; CD4: Cluster of Differentiation 4; CMetS: Cardiometabolic Syndrome; CMet: Cardiometabolic; DBP: Diastolic Blood Pressure; EGIR: European Group for the Study of Insulin Resistance; FBS: Fasting Blood Sugar; HDL-C: High-Density Lipoprotein Cholesterol; HIV: Human Immunodeficiency Virus; IDF: International Diabetes Federation; IGF: Insulin-like Growth Factor; IGT: Impaired Glucose Tolerance; IR: Insulin Resistance; LDL-C: Low-Density Lipoprotein Cholesterol; NCEP: National Cholesterol Education Programme; NHLBI : National Heart, Lung, and Blood Institute; NNRTI: Nonnucleoside Reverse; Transcriptase Inhibitor; NRTI: Nucleoside Reverse Transcriptase Inhibitor; PI: Protease Inhibitor; PLWHA: People Living with HIV-AIDS; PR: Pulse Rate; SBP: Systolic Blood Pressure; TC: Total Cholesterol; TGs: Triglyceride; T2DM: Type 2 Diabetes Mellitus; VL: Viral Load; WHO: World Health Organization; ZMH: Zewditu Memorial Hospital

\section{Declarations}

\section{Ethical statement}

The study was approved by - 1) the Muhimbili University of Health and Allied Sciences, Office of the Director of Research and Publications (Ref. No. 2018-04-23/AEC//Vol. XII/88), Dar el Saalam, Tanzania. 2) Addis Ababa University, School of Pharmacy, Ethical Review Board (ERB/SOP/41/11/2018), Addis Ababa, Ethiopia. 3) Addis Ababa University, College of Health Sciences, Institutional Review Board (IRB, Meeting number 08/2018), Addis Ababa, Ethiopia. 4) City Government of Addis Ababa Health Bureau, Ethical Clearance Committee (Ref no. A/A/HB/344438/227), Addis Ababa, Ethiopia.

\section{Consent of publication}

Not applicable.

\section{Availability of data and materials}

Not applicable.

\section{Competing interests}

The authors declare that they have no competing interests. 


\section{Funding information}

The original funding source for conducting the research was sponsored by DAAD. Part of the research was also sponsored by the EDCTP.

\section{Authors contribution}

MA: Conceptualization, Methodology, Software, Formal analysis, Investigation, Data Curation, Writing - Original Draft, Writing - Review \& Editing, Project administration, Funding acquisition; OM: Supervision, Conceptualization, Methodology, Formal analysis, Investigation, Writing - Review \& Editing; WS: Supervision, Funding acquisition; AS: Data Curation, Writing - Review \& Editing; EE: Supervision, Conceptualization, Methodology, Formal analysis, Investigation, Writing - Review \& Editing

\section{Acknowledgement}

We would like to thank the Muhimbili University of Health and Allied Sciences (MUHAS), Addis Ababa University (AAU), the German Academic Exchanges Services (DAAD), Zewditu memorial Hospital (ZMH), and the European \& Developing Countries Clinical Trials Partnership (EDCTP).

We would like to thank all the research participants in the study. We would also like to forward our appreciation for our research assistants Sr. Tizita Woldeyesus Tadesse, BSc, and Mr Chalachew Teshome Tiruneh, MPH. We are also grateful for statistical resources support from Dr Todd Grande, Dr Jacob Mays, Dr Brandon Foltz, and Dr Mike Crowson.

\section{References}

1. Eaton LA, Kalichman SC. Changes in transmission risk behaviors across stages of HIV disease among people living with HIV. $J$ Assoc Nurses AIDS Care. 2009;20(1):39-49.

2. Wong C, Gange SJ, Moore RD, Justice AC, Buchacz K, Abraham AG, et al. Multimorbidity Among Persons Living with Human Immunodeficiency Virus in the United States. Clin Infect Dis. 2018;66(8):1230-8. doi:10.1093/cid/cix998.

3. Chhoun P, Tuot S, Harries AD, Kyaw NTT, Pal K, Mun P, et al. High prevalence of non-communicable diseases and associated risk factors amongst adults living with HIV in Cambodia. PLoS One. 2017;12(11):e0187591. doi:10.1371/journal.pone.0187591.

4. El-Sadr WM, Holmes CB, Mugyenyi P, Thirumurthy H, Ellerbrock T, Ferris R, et al Scale-up of HIV treatment through PEPFAR: a historic public health achievement. Journal of acquired immune deficiency syndromes (1999). 2012;60(Suppl 3):S96.

5. Haacker M, Bärnighausen T, Atun R. HIV and the growing health burden from noncommunicable diseases in Botswana: modelling study. Journal of global health. 2019;9(1).

6. Todowede 00, Sartorius B. Prevalence of metabolic syndrome, discrete or comorbid diabetes and hypertension in sub-Saharan Africa among people living with HIV versus HIV-negative populations: a systematic review and meta-analysis protocol. BMJ open. 2017;7(7):e016602.

7. Srivastava AK. Challenges in the treatment of cardiometabolic syndrome. Indian Journal of Pharmacology. 2012;44(2):155-6. doi:10.4103/0253-7613.93579.

8. Varriale P, Saravi G. Eliezer Hernandez a, Francisco Carbon. Acute myocardial infarction in patients infected with human immunodeficiency virus American. Heart Journal. 2004;147(1):55-9.

9. Suzanne Willard. The Nurse Practitioner's Role in Managing Dyslipidemia and Other Cardiovascular Risk Factors in HIV-Infected Patients: Impact of Antiretroviral Therapy. JANAC. 2006;17(1).

10. Dalal JJ, Padmanabhan T, Jain P, Patil S, Vasnawala H, Gulati A. LIPITENSION: Interplay between dyslipidemia and hypertension. Indian journal of endocrinology metabolism. 2012;16(2):240.

11. Abebe SM, Getachew A, Fasika S, Bayisa M, Demisse AG, Mesfin N. Diabetes mellitus among HIV-infected individuals in follow-up care at University of Gondar Hospital, Northwest Ethiopia. BMJ open. 2016;6(8):e011175.

12. Mohammed AE, Shenkute TY, Gebisa WC. Diabetes mellitus and risk factors in human immunodeficiency virus-infected individuals at Jimma University Specialized Hospital, Southwest Ethiopia. Diabetes, metabolic syndrome and obesity: targets and therapy. 2015;8:197.

13. Palella FJ Jr, Phair JP. Cardiovascular disease in HIV infection. Curr Opin HIV AIDS. 2011;6(4):266. 
14. Huang PL. A comprehensive definition for metabolic syndrome. Dis Models Mech. 2009;2(5-6):231-7. doi:10.1242/dmm.001180.

15. Moy FM, Bulgiba A. The modified NCEP ATP III criteria maybe better than the IDF criteria in diagnosing Metabolic Syndrome among Malays in Kuala Lumpur. BMC Public Health. 2010;10:678-. doi:10.1186/1471-2458-10-678.

16. Grundy SM, Cleeman JI, Daniels SR, Donato KA, Eckel RH, Franklin BA, et al. Diagnosis and management of the metabolic syndrome: an American Heart Association/National Heart, Lung, and Blood Institute scientific statement. Curr Opin Cardiol. 2006;21(1):1-6.

17. Saltzgiver S, Nielson A, Costello H, Baker A, Chan J, Aguilar D. Dietary Determinants of Metabolic Syndrome Parameters Differ by Gender in College Students. Nutrients. 2019;11(12):2892.

18. Wang M, Shen Q. Advance in metabolic syndrome research. Academic Journal of Second Military Medical University. 1981(03).

19. Sasya M, Devi K, Babu JK, Rayappan B, Bosco J, Krishnan UM. Metabolic Syndrome-An Emerging Constellation of Risk Factors: Electrochemical Detection Strategies. Sensors. 2020;20(1):103.

20. Sa-Caputo DC, Paineiras-Domingos L, Oliveira R, Neves MF, Brandão A, Marin PJ, et al. Acute effects of whole-body vibration on the pain level, flexibility, and cardiovascular responses in individuals with metabolic syndrome. Dose-Response.

2018;16(4):1559325818802139.

21. Strimbu K, Tavel JA. What are biomarkers? Current Opinion in HIV and AIDS. 2010;5(6):463.

22. Mayeux R. Biomarkers: potential uses and limitations. NeuroRx. 2004;1(2):182-8.

23. Hajar R. Evolution of myocardial infarction and its biomarkers: A historical perspective. Heart views: the official journal of the Gulf Heart Association. 2016;17(4):167.

24. Tojek K, Wustrau B, Czerniak B, Korzycka-Wilińska W, Winiarski P, Banaszkiewicz Z, et al. Body mass index as a biomarker for the evaluation of the "Obesity Paradox" among inpatients. Clinical nutrition (Edinburgh. Scotland). 2019;38(1):412-21. doi:10.1016/j.clnu.2017.12.005.

25. Aberg JA, Gallant JE, Anderson J, Oleske JM, Libman H, Currier JS, et al. Primary care guidelines for the management of persons infected with human immunodeficiency virus: recommendations of the HIV Medicine Association of the Infectious Diseases Society of America. Clin Infect Dis. 2004;39(5):609-29.

26. Lamarca K, Garcia Sarasola A, Vidal F, Domingo P. Drug therapies for HIV-related metabolic disorders. Expert opinion on pharmacotherapy. 2016;17(10):1327-38.

27. Lucas GM, Ross MJ, Stock PG, Shlipak MG, Wyatt CM, Gupta SK, et al. Clinical practice guideline for the management of chronic kidney disease in patients infected with HIV: 2014 update by the HIV Medicine Association of the Infectious Diseases Society of America. Clin Infect Dis. 2014;59(9):e96-138.

28. Mengistu N, Azale T, Yimer S, Fikreyesus M, Melaku E, Shumye S. Quality of sleep and associated factors among people living with HIV/AIDS on follow up at Ethiopian Zewditu Memorial Hospital, 2018.

29. Solomon N. Lived experiences of perinatally HIV infected youth; the case of Zewditu memorial hospital; Addis Ababa, Ethiopia. Addis Ababa Universty; 2018.

30. Woldu M, Minzi O, Engidawork E. Prevalence of cardiometabolic syndrome in HIV-infected persons: a systematic review. Journal of Diabetes Metabolic Disorders. 2020. doi:10.1007/s40200-020-00552-x.

31. WHO. Non-communicable diseases and their risk factors The WHO STEPwise approach to noncommunicable disease risk factor surveillance (STEPS) Geneva, Switzerland WHO; 2014.

32. Muhammad J, Jamial MM, Ishak A. Home blood pressure monitoring has similar effects on office blood pressure and medication compliance as usual care. Korean journal of family medicine. 2019;40(5):335.

33. Sutton MSJ, Pfeffer MA, Moye L, Plappert T, Rouleau JL, Lamas G, et al. Cardiovascular death and left ventricular remodeling two years after myocardial infarction: baseline predictors and impact of long-term use of captopril: information from the Survival and Ventricular Enlargement (SAVE) trial. Circulation. 1997;96(10):3294-9.

34. Poundstone KE, Chaisson RE, Moore RD. Differences in HIV disease progression by injection drug use and by sex in the era of highly active antiretroviral therapy. Aids. 2001;15(9):1115-23.

35. Kagay C, Porco T, Liechty C, Charlebois E, Clark R, Guzman D, et al. Modeling the impact of modified directly observed antiretroviral therapy on HIV suppression and resistance, disease progression, and death. Clinical infectious diseases. 2004;38(Supplement_5):414-S20.

36. Odhiambo C, Davis J, Omolo B. Risk for Cardiovascular Disease in Blacks with HIV/AIDS in America: A Systematic Review and Metaanalysis. Journal of Health Disparities Research Practice. 2017;10(2):8.

Page 17/21 
37. Barbaro G, Di Lorenzo G, Cirelli A, Grisorio B, Lucchini A, Hazra C, et al. An open-label, prospective, observational study of the incidence of coronary artery disease in patients with HIV infection receiving highly active antiretroviral therapy. Clinical therapeutics. 2003;25(9):2405-18.

38. Sudano I, Spieker LE, Noll G, Corti R, Weber R, Lüscher TF. Cardiovascular disease in HIV infection. Am Heart J. 2006;151(6):1147-55. doi:https://doi.org/10.1016/j.ahj.2005.07.030.

39. Jacobson DL, Tang AM, Spiegelman D, Thomas AM, Skinner S, Gorbach SL, et al. Incidence of metabolic syndrome in a cohort of HIV-infected adults and prevalence relative to the US population (National Health and Nutrition Examination Survey). JAIDS Journal of Acquired Immune Deficiency Syndromes. 2006;43(4):458-66.

40. Woldu M, Minzi O, Engidawork E. Prevalence of cardiometabolic syndrome in HIV-infected persons: a systematic review. Journal of Diabetes Metabolic Disorders. 2020;19(2):1671-83. doi:10.1007/s40200-020-00552-x.

41. Dimodi HT, Etame LS, Nguimkeng BS, Mbappe FE, Ndoe NE, Tchinda JN, et al. Prevalence of metabolic syndrome in HIV-infected Cameroonian patients. World Journal of AIDS. 2014;2014.

42. Obirikorang C, Quaye L, Osei-Yeboah J, Odame EA, Asare I. Prevalence of metabolic syndrome among HIV-infected patients in Ghana: A cross-sectional study. Nigerian medical journal: journal of the Nigeria Medical Association. 2016;57(2):86.

43. Bosho DD, Dube L, Mega TA, Adare DA, Tesfaye MG, Eshetie TC. Prevalence and predictors of metabolic syndrome among people living with human immunodeficiency virus (PLWHIV). Diabetol Metab Syndr. 2018;10(1):1-9.

44. Hirigo AT, Tesfaye DY. Influences of gender in metabolic syndrome and its components among people living with HIV virus using antiretroviral treatment in Hawassa, southern Ethiopia. BMC Res Notes. 2016;9(1):1-7.

45. Rogalska-Płońska M, Grzeszczuk A, Rogalski P, Łucejko M, Flisiak R. Metabolic syndrome in HIV infected adults in Poland. Kardiologia Polska (Polish Heart Journal). 2018;76(3):548-53.

46. de Saint Martin L, Pasquier E, Roudaut N, Vandhuick O, Vallet S, Bellein V, et al. Metabolic syndrome: a major risk factor for atherosclerosis in HIV-infected patients (SHIVA study). La Presse Médicale. 2008;37(4):579-84.

47. Samaras K, Wand H, Law M, Emery S, Cooper D, Carr A. Prevalence of metabolic syndrome in HIV-infected patients receiving highly active antiretroviral therapy using International Diabetes Foundation and Adult Treatment Panel III criteria: associations with insulin resistance, disturbed body fat compartmentalization, elevated C-reactive protein, and hypoadiponectinemia. Diabetes Care. 2007;30(1):113-9.

48. Choi KM, Kim SM, Kim Y-E, Choi DS, Baik SH, Lee J. Prevalence and cardiovascular disease risk of the metabolic syndrome using National Cholesterol Education Program and International Diabetes Federation definitions in the Korean population. Metabolism. 2007;56(4):552-8. doi:https://doi.org/10.1016/j.metabol.2006.12.003.

49. Lundgren JD, Battegay M, Behrens G, De Wit S, Guaraldi G, Katlama C, et al. European AIDS Clinical Society (EACS) guidelines on the prevention and management of metabolic diseases in HIV. HIV medicine. 2008;9(2):72-81.

50. Nguyen KA, Peer N, Mills EJ, Kengne AP. A meta-analysis of the metabolic syndrome prevalence in the global HIV-infected population. PloS one. 2016;11(3):e0150970.

51. Oguoma V, Nwose EU, Richards R. Prevalence of cardio-metabolic syndrome in Nigeria: a systematic review. Public Health. 2015;129(5):413-23.

52. Zhang Z, Fan S, Xue Z, Yuan J, Zhou Z, Wang T, et al. Evaluation of the appropriate predictive contributor and diagnostic threshold for the cardio-metabolic syndrome in Chinese Uyghur adults. BMC Public Health. 2019;19(1):613. doi:10.1186/s12889-019-6803-4.

53. Cahn P, Leite O, Rosales A, Cabello R, Alvarez C, Seas C, et al. Metabolic profile and cardiovascular risk factors among Latin American HIV-infected patients receiving HAART. Brazilian Journal of Infectious Diseases. 2010;14(2):158-66.

54. Alvarez C, Salazar R, Galindez J, Rangel F, CastaÃ \pm eda M, Lopardo G, et al. Metabolic syndrome in HIV-infected patients receiving antiretroviral therapy in Latin America. Brazilian Journal of Infectious Diseases. 2010;14(3):256-63.

55. Berhane T, Yami A, Alemseged F, Yemane T, Hamza L, Kassim M, et al. Prevalence of lipodystrophy and metabolic syndrome among HIV positive individuals on Highly Active Anti-Retroviral treatment in Jimma, South West Ethiopia. Pan African Medical Journal. 2012;13(1).

56. Mashinya F, Alberts M, Colebunders R. Assessment of cardiovascular risk factors in people with HIV infection treated with ART in rural South Africa: a cross sectional study. AIDS research therapy. 2015;12(1):42.

57. Achwoka D, Waruru A, Chen T-H, Masamaro K, Ngugi E, Kimani M, et al. Noncommunicable disease burden among HIV patients in care: a national retrospective longitudinal analysis of HIV-treatment outcomes in Kenya, 2003-2013. BMC Public Health.

2019;19(1):372. 
58. Dimock D, Thomas V, Cushing A, Purdy JB, Worrell C, Kopp JB, et al. Longitudinal assessment of metabolic abnormalities in adolescents and young adults with HIV-infection acquired perinatally or in early childhood. Metabolism. 2011;60(6):874-80.

59. Sánchez Torres AM, Munoz Muniz R, Madero R, Borque C, García-Miguel MJ, De José Gómez MI. Prevalence of fat redistribution and metabolic disorders in human immunodeficiency virus-infected children. Eur J Pediatr. 2005;164(5):271-6. doi:10.1007/s00431-0041610-y.

60. Manner IW, Trøseid M, Oektedalen O, Baekken M, Os I. Low nadir CD4 cell count predicts sustained hypertension in HIV-infected individuals. The Journal of Clinical Hypertension. 2013;15(2):101-6.

61. Després J-P. Abdominal obesity: the most prevalent cause of the metabolic syndrome and related cardiometabolic risk. European Heart Journal Supplements. 2006;8(suppl_B):B4-12.

62. Nilsson PM, Laurent S, Cunha PG, Olsen MH, Rietzschel E, Franco OH, et al. Characteristics of healthy vascular ageing in pooled population-based cohort studies: the global Metabolic syndrome and Artery REsearch Consortium. Journal of hypertension. 2018;36(12):2340.

63. Locher JL, Vickers KS, Buys DR, Ellis A, Lawrence JC, Newton LE, et al. A randomized controlled trial of a theoretically-based behavioral nutrition intervention for community elders: lessons learned from the behavioral nutrition intervention for community elders study. Journal of the Academy of Nutrition Dietetics. 2013;113(12):1675-82.

64. Wang H, Naghavi M, Allen C, Barber RM, Bhutta ZA, Carter A, et al. Global, regional, and national life expectancy, all-cause mortality, and cause-specific mortality for 249 causes of death, 1980-2015: a systematic analysis for the Global Burden of Disease Study 2015. The lancet. 2016;388(10053):1459-544.

65. Suglia SF, Koenen KC, Boynton-Jarrett R, Chan PS, Clark CJ, Danese A, et al. Childhood and adolescent adversity and cardiometabolic outcomes: a scientific statement from the American Heart Association. Circulation. 2018;137(5):e15-28.

66. McCutchan J, Marquie-Beck J, Fitzsimons C, Letendre S, Ellis R, Heaton R, et al. Role of obesity, metabolic variables, and diabetes in HIV-associated neurocognitive disorder. Neurology. 2012;78(7):485-92.

67. Kwiatkowska W, Knysz B, Drelichowska-Durawa J, Czarnecki M, Gasiorowski J, Biłyk E, et al. Overweight, obesity and underweight in HIV infected patients. Przeglad lekarski. 2013;70(3):113.

68. Mutimura E, Crowther NJ, Stewart A, Todd Cade W. The human immunodeficiency virus and the cardiometabolic syndrome in the developing world: an African perspective. Journal of the cardiometabolic syndrome. 2008;3(2):106-10.

69. Arrive E, Viard J-P, Salanave B, Dollfus C, Matheron S, Reliquet V, et al. Metabolic risk factors in young adults infected with HIV since childhood compared with the general population. PloS one. 2018;13(11):e0206745.

70. Van Rooyen J, Kruger H, Huisman H, Wissing M, Margetts B, Venter C, et al. An epidemiological study of hypertension and its determinants in a population in transition: the THUSA study. J Hum Hypertens. 2000;14(12):779-87.

71. Hadigan C, Meigs JB, Corcoran C, Rietschel P, Piecuch S, Basgoz N, et al. Metabolic abnormalities and cardiovascular disease risk factors in adults with human immunodeficiency virus infection and lipodystrophy. Clin Infect Dis. 2001;32(1):130-9.

72. Grundy SM. Hypertriglyceridemia, insulin resistance, and the metabolic syndrome. The American journal of cardiology. 1999;83(9):25-9.

73. Drager LF, Lopes HF, Maki-Nunes C, Trombetta IC, Toschi-Dias E, Alves MJN, et al. The impact of obstructive sleep apnea on metabolic and inflammatory markers in consecutive patients with metabolic syndrome. PloS one. 2010;5(8):e12065.

74. Waters DD, Hsue PY. Lipid abnormalities in persons living with HIV infection. Can J Cardiol. 2019;35(3):249-59.

75. Kotler DP. HIV and antiretroviral therapy: lipid abnormalities and associated cardiovascular risk in HIV-infected patients. JAIDS Journal of Acquired Immune Deficiency Syndromes. 2008;49:79-85.

76. Srinivasa S, Grinspoon SK. Metabolic and body composition effects of newer antiretrovirals in HIV-infected patients. Eur J Endocrinol. 2014;170(5):R185-202.

77. Pinto DS, da Silva MJLV. Cardiovascular disease in the setting of human immunodeficiency virus infection. Current Cardiology Reviews. 2018;14(1):25-41.

78. Offor O, Utay N, Reynoso D, Somasunderam A, Currier J, Lake J. Adiponectin and the steatosis marker Chi3L1 decrease following switch to raltegravir compared to continued PI/NNRTI-based antiretroviral therapy. PloS one. 2018;13(5):e0196395.

79. Mirza F, Luthra P, Chirch L. Endocrinological aspects of HIV infection. J Endocrinol Invest. 2018;41(8):881-99.

80. Friis-Møller N, Weber R, Reiss P, Thiébaut R, Kirk O, Monforte AdA, et al. Cardiovascular disease risk factors in HIV patientsassociation with antiretroviral therapy. Results from the DAD study. Aids. 2003;17(8):1179-93. 
81. Muhammad S, Sani MU, Okeahialam BN. Cardiovascular disease risk factors among HIV-infected Nigerians receiving highly active antiretroviral therapy. Nigerian medical journal: journal of the Nigeria Medical Association. 2013;54(3):185.

82. Rodriguez-Penney AT, ludicello JE, Riggs PK, Doyle K, Ellis RJ, Letendre SL, et al. Co-morbidities in persons infected with HIV: increased burden with older age and negative effects on health-related quality of life. AIDS Patient Care STDs. 2013;27(1):5-16.

83. Tripathi A, Liese AD, Winniford MD, Jerrell JM, Albrecht H, Rizvi AA, et al. Impact of Clinical and Therapeutic Factors on Incident Cardiovascular and Cerebrovascular Events in a Population-Based Cohort of HIV-Infected and Non-HIV-Infected Adults. Clinical cardiology. 2014;37(9):517-22.

\section{Figures}

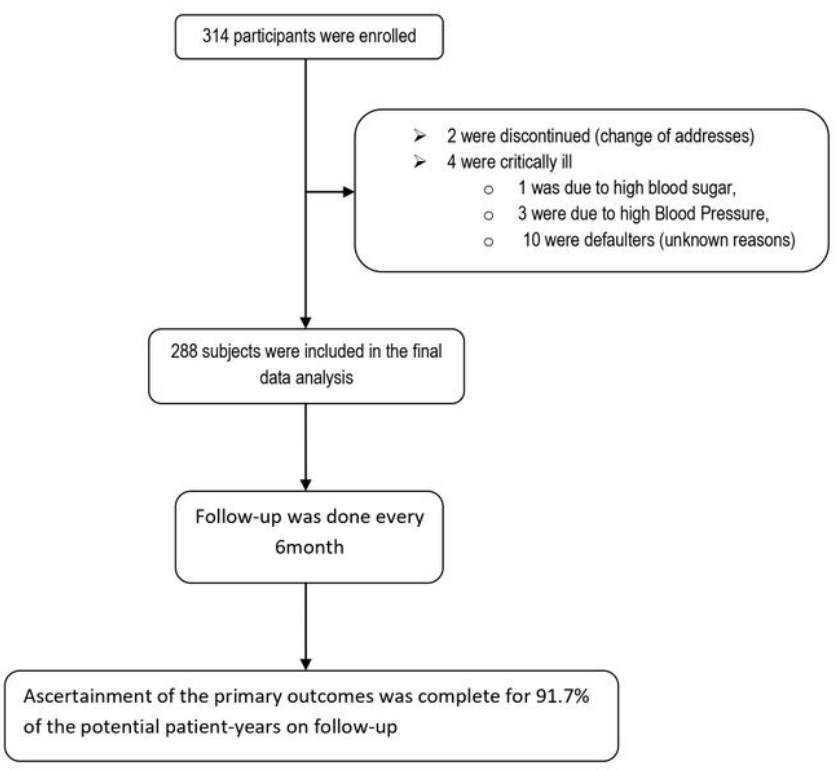

Figure 1 Enrollment, Screening, and Follow-up.

\section{Figure 1}

Enrollment, Screening, and Follow-up 


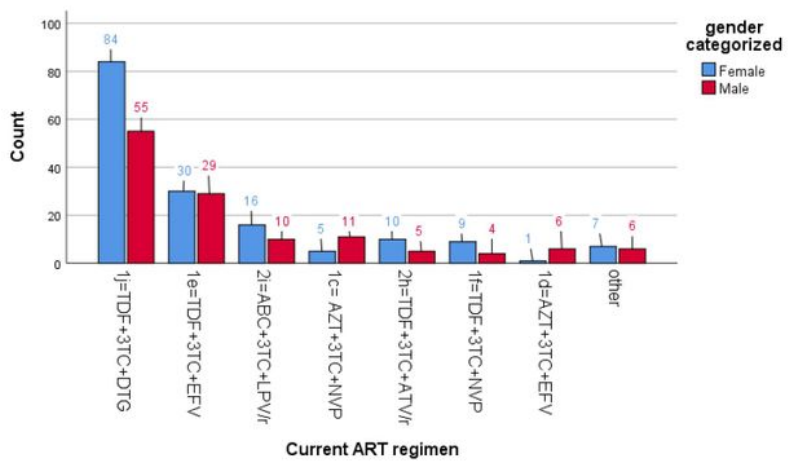

Figure 2 Pattern of current ART regimen based on gender distribution among participants enrolled in the study, Zewditu Memorial Hospital, June 2020, Addis Ababa, Ethiopia.

*Others: $2 \mathrm{f}=\mathrm{AZT}+3 \mathrm{TC}+\mathrm{ATV} / \mathrm{r}(1.7 \%) ; 2 \mathrm{~g}=\mathrm{TDF}+3 \mathrm{TC}+\mathrm{LPV} / \mathrm{r}(1 \%) ; 2 \mathrm{e}=\mathrm{TDF}+3 \mathrm{TC}+\mathrm{ATV} / \mathrm{r}(0.7 \%)$; $3 \mathrm{~b}=\mathrm{DRV} / \mathrm{r}+\mathrm{DTG}+\mathrm{TDF}+3 \mathrm{TC}(0.7 \%) ; \mathrm{l}=\mathrm{ABC}+3 \mathrm{TC}+\mathrm{NVP}(0.3 \%)$

$\mathrm{ABC}=$ Abacavir $\mathrm{ATV} / \mathrm{r}=;$ Atazanavir + ritonavir $; \mathrm{AZT}=$ Zidovudine $; \mathrm{DRV} / \mathrm{r} ;$ Danuravir + ritonavir; $\mathrm{DTG}$; Dolutegravir; $\mathrm{EFV}=\mathrm{Efavirenz} ; \mathrm{LPV} / \mathrm{r}=\mathrm{L}$ Lopinavir + ritonavir; $\mathrm{NVP}=\mathrm{Nevirapin} ; \mathrm{TDF}=\mathrm{Tenofovi}$; 3TC= Lamivudine;

\section{Figure 2}

Pattern of current ART regimen

\section{Supplementary Files}

This is a list of supplementary files associated with this preprint. Click to download.

- Annex.pdf 